\title{
Experiencia en el tratamiento de la estenosis de la unión ureterovesical en pacientes con trasplante renal
}

\section{Experience in the treatment of ureterovesical junction stricture in patients with kidney transplant}

\author{
Hugo Rivera-Astorga, ${ }^{1}$ Harvey Mauricio Medrano-Urtecho, ${ }^{1}$ Luis García-Covarrubias, ${ }^{2}$ Héctor Hinojosa-Heredia, ${ }^{2}$ \\ Ángel Gurrola-Ortega, ${ }^{1}$ Roberto Carlos Sarabia-Estrada, ${ }^{1}$ Leopoldo Mateo Garduño-Arteaga, ${ }^{1}$ Juan Eduardo \\ Sánchez-Núñez, ${ }^{1}$ Christian Cancino-Cubias ${ }^{1}$
}

\section{Resumen}

OBJETIVO: Reportar la experiencia en el tratamiento de la estenosis de la unión ureterovesical en pacientes con trasplante renal.

MATERIALES Y MÉTODOS: Estudio ambispectivo y descriptivo, efectuado en pacientes con estenosis de la unión ureterovesical que recibieron trasplante renal, atendidos en los servicios de Urología y de Trasplantes del Hospital General de México Dr. Eduardo Liceaga, entre los años 2013 a 2018. Se analizaron variables como: edad, tiempo transcurrido a partir del trasplante, permanencia de la nefrostomía, tiempo de permanencia de la estenosis hasta la curación; determinación de creatinina pre y posquirúrgica, permanencia del cateter doble J, seguimiento y tipo de tratamiento establecido. Para el análisis de los datos se utilizó estadística descriptiva (media, mediana, desviación estándar y rangos) con el programa SPSS 17.

RESULTADOS: Se registraron 4 casos con estenosis de la unión ureterovesical: 2 se trataron con dilatación endoscópica con balón y 2 mediante ureterotomía con corte frio y láser. A tres pacientes se les practicó nefrostomía con la finalidad de preservar la función renal y el catéter se retiró, en promedio, 3 meses después de su colocación. Hasta el momento todos los pacientes se encuentran sin evidencia de reestenosis, a 36 meses de seguimiento.

CONCLUSIONES: Las distintas modalidades de tratamiento endoscópico son efectivas y seguras en pacientes con estenosis de la anastomosis ureterovesical a corto y mediano plazos; no obstante, debe individualizarse cada caso, porque hasta el momento no existe un consenso que establezca cuál es la mejor técnica en este tipo de complicación.

PALABRAS CLAVE: Trasplante renal; estenosis ureteral; complicaciones urológicas.

\section{Abstract}

OBJECTIVE: To report our experience in the management of ureterovesical stricture in transplanted patients.

MATERIALS AND METHODS: An ambispective and descriptive study was conducted on patients with ureterovesical junction stricture that were kidney transplantation recipients, seen at the Urology and Transplantation Services of the Hospital General de México Dr. Eduardo Liceaga within the time frame of 2013 to 2018. The variables analyzed were: age, time since transplantation, time with nephrostomy, time from stricture to correction, preoperative and postoperative creatinine levels, length of time with doubleJ catheter, follow-up, and type of treatment established. Descriptive statistics (mean, median, standard deviation, and range) were used for the data analysis, employing the SPSS 17 program.

RESULTS: Four cases of ureterovesical junction stricture were registered: 2 were treated with endoscopic balloon dilation and 2 through cold knife and laser ureterotomy. At

\footnotetext{
${ }^{1}$ Servicio de Urología.

Servicio de Trasplantes.
}

Hospital General de México Dr. Eduardo Liceaga, Ciudad de México.

Recibido: junio 2018

Aceptado: julio 2018

Correspondencia Hugo Rivera Astorga hugolch_9@hotmail.com

Este artículo debe citarse como Rivera-Astorga $\mathrm{H}$, Medrano-Urtecho HM, García-Covarrubias L, Hinojosa Heredia H, Gurrola-Ortega A, SarabiaEstrada RC, Garduño-Arteaga LM, Sánchez-Núñez JE, , Cancino-Cubias C. Experiencia en el tratamiento de la estenosis de la unión ureterovesical en pacientes con trasplante renal. Rev Mex Urol. 2018 julio-agosto;78(4): 290-297. DOI: https://doi.org/10.24245/revmexurol.v78i4.2341 
present, after 36 months of follow-up, none of the patients have presented with signs of re-stricture.

CONCLUSIONS: The different endoscopic treatment modalities are safe and effective in patients with stricture of the ureterovesical anastomosis in the short term and the medium term. However, each case must be individualized, given that at present there is no consensus on the best technique for that type of complication.

KEYWORDS: Kidney transplantation; Ureteral stricture; Urologic complications.

\section{ANTECEDENTES}

La incidencia de complicaciones urológicas en pacientes con trasplante renal varía de 2 a $3 \%{ }^{1}$ Hasta la fecha no existen guías o consensos de tratamiento estandarizados de las complicaciones urológicas en pacientes con trasplante renal; la mayor parte de los estudios comprende reportes o pequeñas series de casos. ${ }^{2}$

Las complicaciones urológicas asociadas frecuentemente con trasplante renal incluyen: reflujo vesicoureteral $(2-86 \%),{ }^{2,3}$ urolitiasis en el sitio del injerto $(0.4-1 \%),{ }^{3-6}$ disfunción de vaciamiento vesical postrasplante $(1.1 \%),{ }^{7}$ estenosis de la unión ureterovesical $(2-7.5 \%),{ }^{8}$ fuga o incontinencia urinaria $(1.2-8.9 \%) .^{2}$

La estenosis ureteral es una de las complicaciones más frecuentes en pacientes con trasplante renal; el segmento más comúnmente afectado es la anastomosis ureterovesical, cuya incidencia varía de $1-4.5 \% .{ }^{9}$ Esta alteración resulta de la pérdida de vascularización ureteral, obstrucción extrínseca (linfocele, fibrosis) o deficiencia en la técnica quirúrgica. Entre los factores de riesgo se incluyen: edad del donante (mayor de 65 años), variantes anatómicas vasculares (más de dos arterias renales en el aloinjerto), tiempo de isquemia fría prolongada y técnica anastomótica sin stent. ${ }^{10}$ La modalidad de adquisición renal (mediante cirugía abierta o laparoscópica) y la técnica de implantación (intra vs extravesical) no se han relacionado con la formación de estenosis de la unión ureterovesical. ${ }^{10} \mathrm{El}$ deterioro asinto- mático de la función renal es la manifestación más común. La lesión renal suele originarse tempranamente después de la remoción del catéter ureteral doble J; incluso los pacientes a quienes no se les coloca catéter ureteral doble J durante la anastomosis suelen manifestar deterioro de la función renal de manera más temprana. El diagnóstico se establece con ultrasonido o tomografía, esta última con mayor ventaja de visualizar todo el trayecto ureteral e identificar fuentes extrínsecas e intrínsecas de obstrucción. En ambos estudios de imagen la dilatación ureteropielocalicial es el hallazgo principal para establecer el diagnóstico presuntivo. Las técnicas endourológicas se han utilizado para descomprimir el sistema colector antes de realizar cualquier procedimiento abierto para la reconstrucción ureteral. La colocación de un catéter ureteral de manera retrógrada representa un desafío médico y fuente potencial de peligro; por tanto, algunos investigadores consideran que la colocación de nefrostomía es la técnica de primera línea en el tratamiento de esta alteración.

En fechas recientes se ha posicionado el tratamiento endourológico como el protocolo inicial y entre las opciones endoscópicas se incluyen: dilatación con balón o endoureterectomía, que pueden realizarse con bisturí en frío, catéter de corte con globo o láser de Holmio, incluso con técnica anterógrada o retrógrada. Pese a ello, si se considera la porción dilatada como la región de mejor acceso, entonces el acceso anterógrado resultará más accesible. Una investigación que evaluó la dilatación con balón primario, efec- 
tuada en 94 pacientes ${ }^{8,11-13}$ con seguimiento de 37.3 meses (límites de 17 a 78 meses), reportó una tasa de éxito de $51 \%$ (límites de 44 a $62 \%$ ) y la reintervención con dilatación fue efectiva en $25 \%$ de los casos. ${ }^{5}$ Otra serie de casos encontró que la dilatación en los primeros 3 meses del trasplante se asoció con una tasa de éxito de 74 vs $44 \% .^{11}$

El dispositivo de corte de balón Acucise ${ }^{\circledR}$ se introdujo por primera vez en 1993 y se ha utilizado para el tratamiento de la estenosis de la unión ureteropiélica. Algunos estudios reportan su uso en pacientes con estenosis de la unión ureterovesical después del trasplante renal. ${ }^{8,14-16} \mathrm{En}$ una serie de 21 pacientes, tratados con dilatación con balón (promedio de seguimiento de 19 meses), se reportó una tasa de éxito de $60-100 \%$. A pesar de las tasas de éxito razonables, la técnica se ha descontinuado debido a la hemorragia relacionada con la incisión ureteral.

La endoureterectomía con visión directa se ha convertido en el tratamiento de elección, pues resulta más efectiva en la dilatación con balón y la incisión ureteral es más segura que la realizada en forma ciega. La endoureterectomía puede efectuarse con corte frío, electrocauterio o láser de Holmio, con una tasa de éxito de $63-100 \%$ a 30 meses de seguimiento; ; $17-20$ no obstante, los ensayos se han efectuado con muestras poblacionales pequeñas y heterogéneas. Tres estudios que evaluaron las técnicas anterógrada y retrógrada evidenciaron como complicación más común la estenosis de la anastomosis ureterovesical y, aunque el tratamiento se efectúa por acceso abierto, la modalidad endourológica puede realizarse en pacientes seleccionados (manifestaciones clínicas tempranas, obstrucción parcial y estenosis distal menor de $1 \mathrm{~cm}$ ). Hoy día, la endoureterectomía con visión directa representa la técnica preferida de la mayoría de los médicos. ${ }^{18,20}$
El objetivo de este estudio es reportar la experiencia en el tratamiento de la estenosis de la unión ureterovesical en pacientes con trasplante renal.

\section{MATERIALES Y MÉTODOS}

Estudio ambispectivo y descriptivo, efectuado en pacientes con estenosis de la unión ureterovesical que recibieron trasplante renal, atendidos en los servicios de Urología y de Trasplantes del Hospital General de México Dr. Eduardo Liceaga, entre los años 2013 a 2018. Se analizaron variables como: edad, tiempo transcurrido a partir del trasplante, permanencia de la nefrostomía, tiempo de la estenosis hasta la curación; determinación de creatinina pre y posquirúrgica, permanencia del cateter doble J, seguimiento y tipo de tratamiento establecido.

Para el análisis de los datos se utilizó estadística descriptiva (media, mediana, desviación estándar y rangos) con el programa SPSS 17.

\section{RESULTADOS}

Durante el periodo de estudio se realizaron 200 trasplantes renales. Se identificaron 4/200 casos de estenosis de la unión ureterovesical: dos hombres y dos mujeres, con promedio de edad de 35.7 años. Dos casos se trataron con dilatación endoscópica con balón, uno mediante ureterotomía con corte frio y el otro con láser de Holmium. A tres pacientes se les practicó nefrostomía con la finalidad de preservar la función renal y el catéter se retiró, en promedio, 3 meses después de su colocación. El caso 4 decidió que no se le colocara la derivación; sin embargo, se efectuó cirugía resolutiva 15 días después de establecer el diagnóstico. No se reportaron complicaciones asociadas con el procedimiento.

En todos los casos se colocó un catéter doble J posterior a la cirugía, con promedio de per- 
Rivera-Astorga $\mathrm{H}$ y col. Estenosis de la unión ureterovesical en trasplante renal

manencia de 5 semanas. Hasta el momento se encuentran sin evidencia de reestenosis y con adecuada función renal, a 36 meses de seguimiento (Cuadro 1).

A continuación se describen los casos de estenosis de la unión ureterovesical identificados en el periodo de estudio:

\section{Caso 1}

Paciente femenina de 29 años de edad, con trasplante renal de donador vivo relacionado, de un año de evolución, quien acudió a consulta por dolor en la fosa iliaca derecha. A su ingreso, los estudios de laboratorio reportaron concentraciones elevadas de creatinina sérica; el ultrasonido

Cuadro 1. Características de los pacientes con estenosis de la unión ureterovesical postrasplante

\begin{tabular}{|c|c|c|c|c|c|}
\hline Variable & Caso 1 & Caso 2 & Caso 3 & Caso 4 & Media \\
\hline Edad (años) & 29 & 32 & 46 & 36 & 35.7 \\
\hline $\begin{array}{l}\text { Tiempo transcurrido a partir } \\
\text { del trasplante (meses) }\end{array}$ & 12 & 24 & 24 & 12 & 18 \\
\hline $\begin{array}{l}\text { Permanencia de la nefrostomía } \\
\text { (meses) }\end{array}$ & 4 & 3 & 2 & - & 3 \\
\hline $\begin{array}{l}\text { Tiempo de permanencia de la } \\
\text { estenosis a la curación (meses) }\end{array}$ & 5 & 4 & 3 & $1 / 2$ & 3 \\
\hline Creatinina al ingreso (mg/dL) & 1.8 & 2.2 & 1.6 & 3.2 & 2.2 \\
\hline $\begin{array}{l}\text { Creatinina posquirúrgica (mg/ } \\
\mathrm{dL} \text { ) }\end{array}$ & 1.5 & 2.0 & 1.5 & 2.2 & 1.8 \\
\hline $\begin{array}{l}\text { Permanencia del catéter doble } \\
\text { J ( semanas) }\end{array}$ & 8 & 3 & 6 & 3 & 5 \\
\hline Seguimiento (meses) & 36 & 30 & 26 & 20 & 28 \\
\hline Tratamiento & Balón & Corte frío & Balón & Láser & - \\
\hline
\end{tabular}

mostró ectasia del riñón trasplantado, por lo que se decidió la nefrostomía. La nefrostografía evidenció progresión del medio de contraste hasta la unión ureterovesical sin paso a la vejiga. Se practicó dilatación endoscópica de la estenosis con balón y se colocó un catéter ureteral doble J. Figuras 1 y 2.

\section{Caso 2}

Paciente masculino de 32 años que recibió trasplante renal de donador vivo relacionado, de 2 años de evolución. El paciente acudió a revisión de rutina, sin manifestación de síntomas clínicos. El análisis de laboratorio reportó creatinina

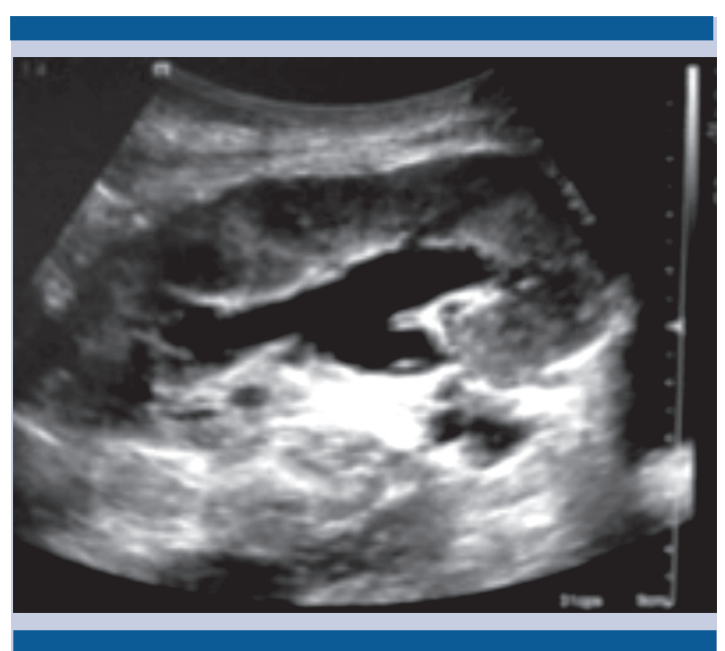

Figura 1. Riñón trasplantado con ectasia. 


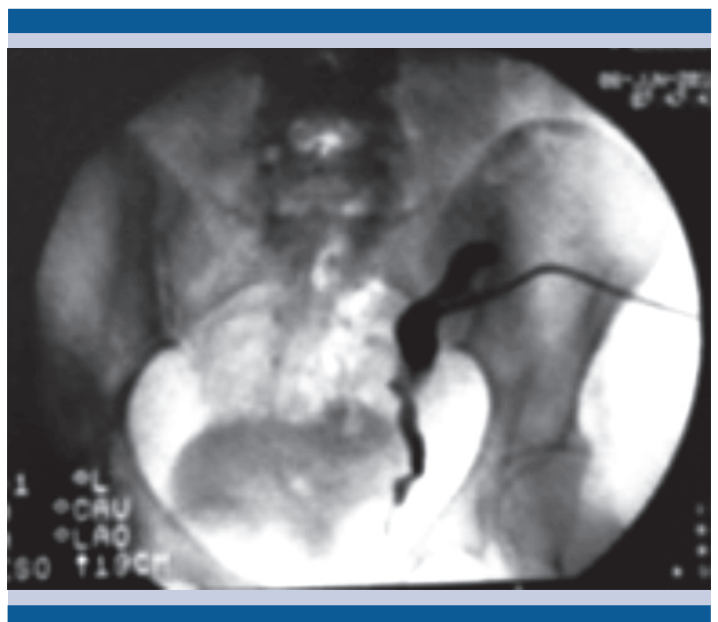

Figura 2. Nefrostografía de riñón trasplantado, sin paso del medio de contraste hacia la vejiga; solo se observa el paso a la unión ureterovesical.

sérica elevada; el ultrasonido y la tomografía mostraron ectasia renal, por lo que se colocó la nefrostomía. Al igual que el caso anterior, no se apreció el paso del medio de contraste a la vejiga, con pérdida de la progresión en la unión ureterovesical. El tratamiento consistió en ureterotomía con corte frío y colocación de un catéter ureteral doble J. Figuras $\mathbf{3 , 4}$ y $\mathbf{5}$.

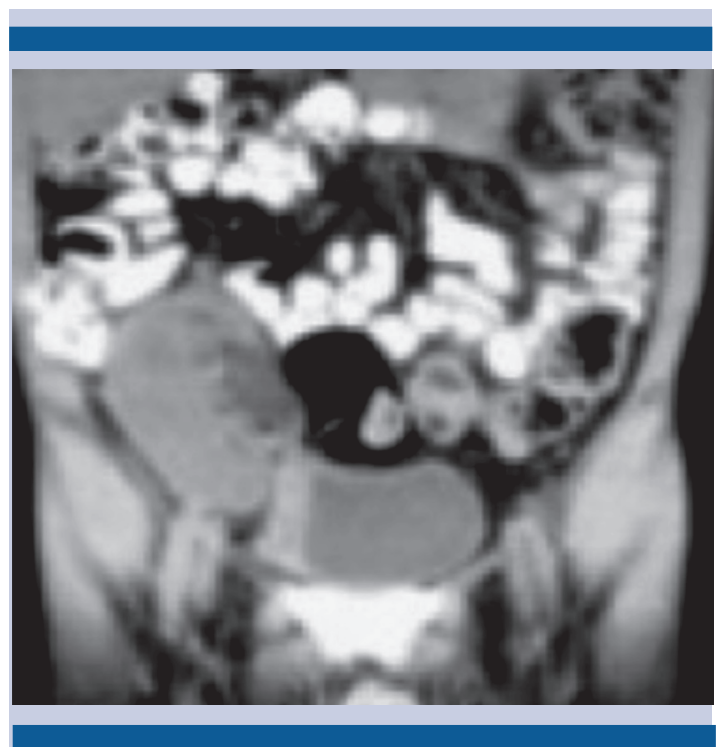

Figura 3. Tomografía que muestra el riñón trasplantado con dilatación ureteropielocalicial.

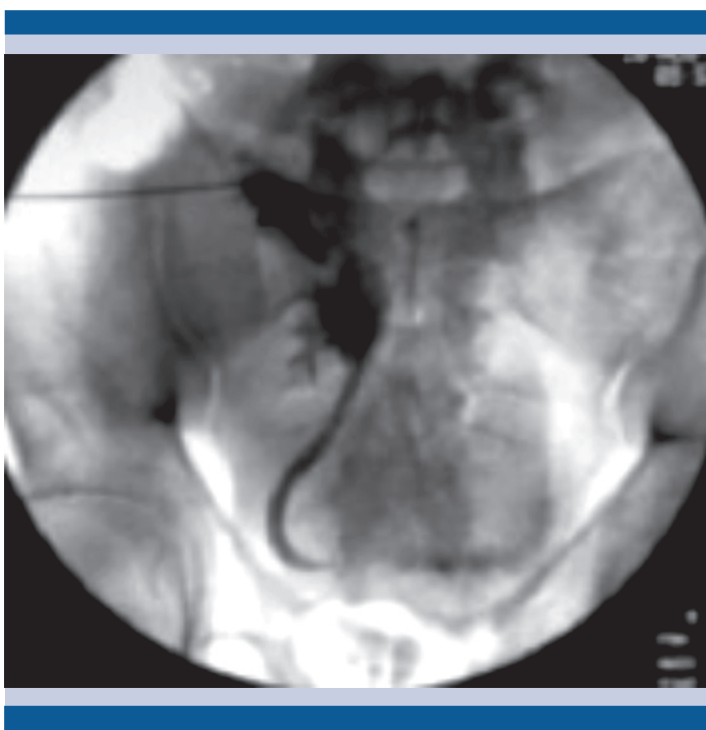

Figura 4. Nefrostografía del riñón trasplantado sin paso del medio de contraste hacia la vejiga.

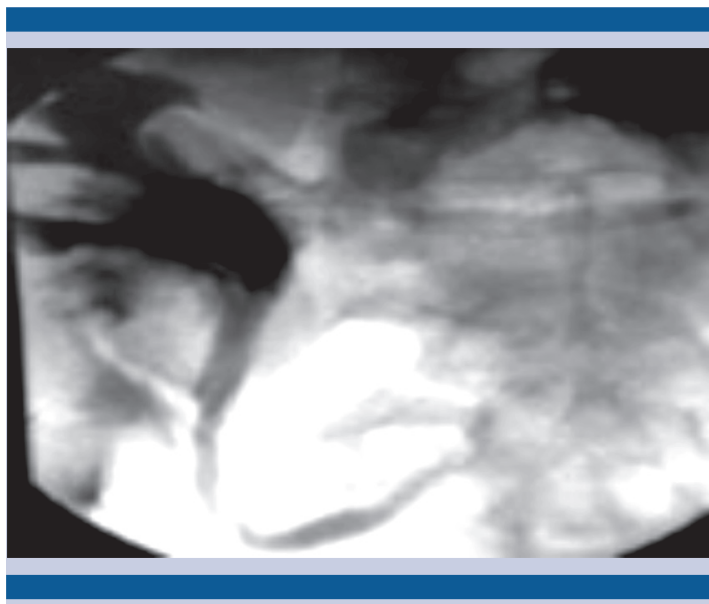

Figura 5. Nefrostografía sin paso del medio de contraste hacia la vejiga y con dilatación pielocalicial.

\section{Caso 3}

Paciente masculino de 46 años de edad, con trasplante renal de donador vivo relacionado, quien acudió al servicio médico por dolor en la fosa iliaca derecha e hipogastrio. A su ingreso tuvo concentración elevada de creatinina y fiebre. La urotomografia no mostró el paso del medio de 
Rivera-Astorga $\mathbf{H}$ y col. Estenosis de la unión ureterovesical en trasplante renal

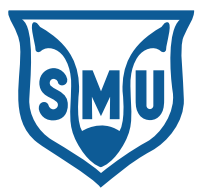

contraste a la vejiga, ni su progresión en la unión ureterovesical; sin embargo, se observó dilatación ureteropielocalicial importante. Se practicó nefrostomía de urgencia, se realizó la dilatación endoscópica con balón y la colocación de un catéter doble J. Figuras 6 y 7.

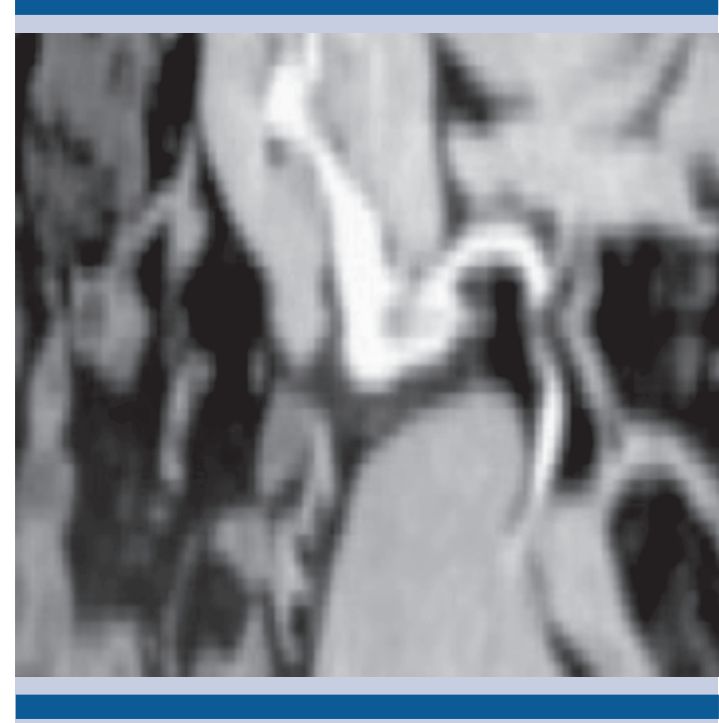

Figura 6. Tomografía con fase de eliminación que no evidencia el paso del medio de contraste hacia la vejiga.

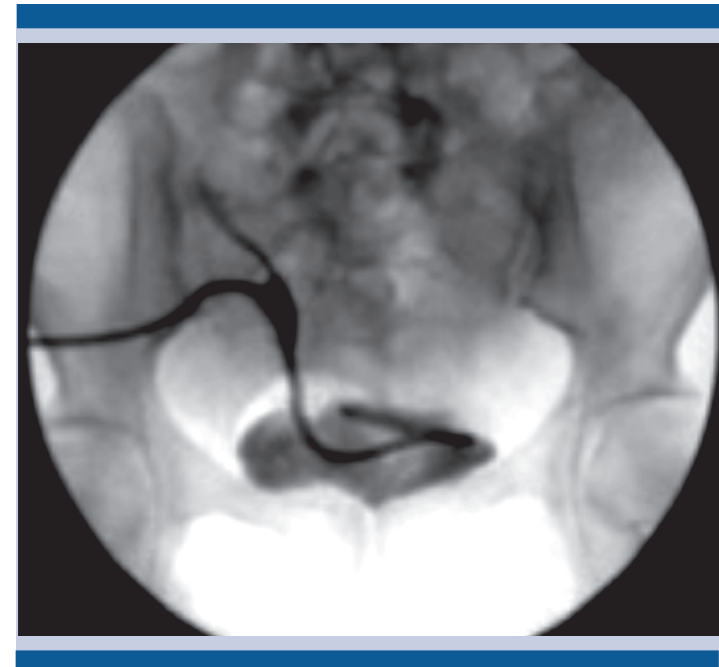

Figura 7. Nefrostografía que muestra el paso de la nefrostomía hasta la cavidad vesical ("cola de cochino" en la cavidad vesical).
Caso 4

Paciente femenina de 36 años, con trasplante renal de donador vivo relacionado, quien acudió a consulta por dolor en el hueco pélvico y elevación de azoados. El ultrasonido y la tomografía mostraron dilatación de las cavidades del riñón trasplantado. Se realizó ureterotomía con láser Ho-YAG y se le colocó un catéter J. Figura 8.

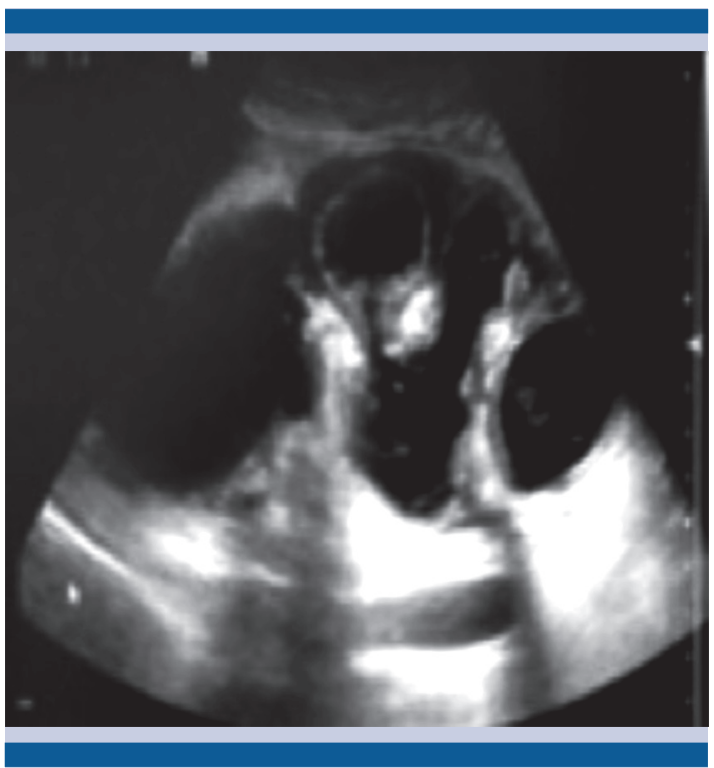

Figura 8. Riñón trasplantado con ectasia.

\section{DISCUSIÓN}

La estenosis de la anastomosis ureterovesical representa una de las complicaciones urológicas más frecuentes del trasplante renal; ${ }^{8}$ sin embargo, hasta el momento no existe un consenso de tratamiento estandarizado. Actualmente, la cirugía abierta (reimplante ureteral) proporciona la mayor tasa de éxito, ${ }^{22}$ incluso algunos procedimientos endoscópicos también ofrecen tasas de éxito considerables, pero con esta complicación; sin embargo, aún no existe suficiente evidencia para su estandarización. 
Aunque el número de casos (estenosis ureterovesical) atendidos en nuestro servicio no es muy grande, hemos empleado diversos procedimientos endoscópicos (dilatación con balón, corte frio con cuchilla y láser Ho-YAG). para su tratamiento y curación.

Al igual que en otros centros de trasplante, no se tiene establecido un tratamiento de referencia para la complicación asociada con el trasplante renal; por tanto, cada caso debe individualizarse. Los resultados clínicos y radiológicos posoperatorios reportados en nuestros pacientes son satisfactorios, ya que los estudios de imagen de control no evidencian dilatación ureteropielocalicial y la concentración de creatinina sérica se encuentra dentro de los parámetros normales.

\section{Figura 9}

En cuanto a la dilatación con balón, nuestros resultados coinciden con lo reportado en la bibliografía, que reportan tasas de éxito de $78 \% .^{11-13}$

El corte frio con bisturí ha demostrado similar eficacia que la dilatación endoscópica con balón. ${ }^{19}$ El paciente que recibió tratamiento

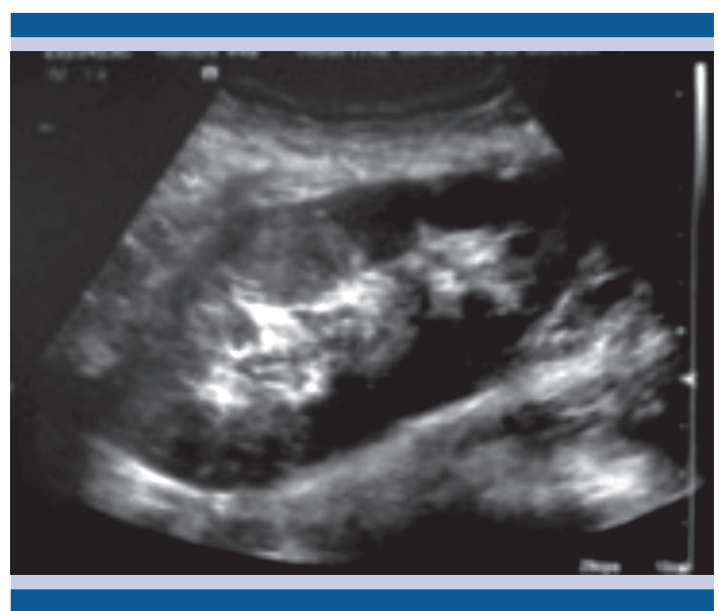

Figura 9. Ultrasonido de control sin evidencia de ectasia renal posterior a la corrección de la estenosis de la unión ureterovesical. con láser Ho-YAG tuvo curación completa a 20 meses de seguimiento, que igualmente coincide con diversas investigaciones, que reportan éxito de $79 \%(63-100 \%){ }^{18,20,21}$

\section{CONCLUSIONES}

Las distintas modalidades de tratamiento endourológico son efectivas y seguras en pacientes con estenosis de la anastomosis ureterovesical a corto y mediano plazos; no obstante, es importante individualizar cada caso, porque hasta el momento no existe un consenso que establezca cuál es la mejor técnica quirúrgica endoscópica en este tipo de complicación.

Se requieren estudios adicionales, con diseño metodológico distinto y mayor número de muestra, con la finalidad de aportar evidencia fehaciente de la mejor alternativa terapéutica en pacientes con estenosis de la anastomosis ureterovesical que reciben trasplante renal.

\section{REFERENCIAS}

1. Streeter EH, et al. The urological complications of renal transplantation: a series of 1535 patients. BJU Int 2002;90(7):627-634.

2. Pichler R, et al. Endoscopic application of dextranomer/hyaluronic acid copolymer in the treatment of vesico-ureteric reflux after renal transplantation. BJU Int 2001;107(12):1967-1972.

3. Favi $\mathrm{E}$, et al. Long-term clinical impact of vesicoureteral reflux in kidney transplantation. DOI: https://doi. org/10.1016/j.transproceed.2009.03.052

4. Mastrosimone $\mathrm{S}$, et al. Clinical significance of vesicoureteral reflux after kidney transplantation. Clin Nephrol 1993;40(1):38-45.

5. Stenberg A, Lackgren G. A new bioimplant for the endoscopic treatment of vesicoureteral reflux: experimental and short-term clinical results. J Urol 1995;154(2):800-803.

6. Elder JS, et al. Endoscopic therapy for vesicoureteral reflux: a meta-analysis. I. reflux resolution and urinary tract infection. DOI: https://doi.org/10.1016/S00225347(05)00210-7

7. Yucel S, et al. Endoscopic vesicoureteral reflux correction in transplanted kidneys: does injection technique matter? DOI: https://doi.org/10.1089/end.2010.0219 
Rivera-Astorga $\mathbf{H}$ y col. Estenosis de la unión ureterovesical en trasplante renal

8. Juaneda B, et al. Endourological management is better in early-onset ureteral stenosis in kidney transplantation. DOI: https://doi.org/10.1016/j.transproceed.2005.09.199

9. Mano $\mathrm{R}$, et al. Retrograde endoureterotomy for persistent ureterovesical anastomotic strictures in renal transplant kidneys after failed antegrade balloon dilation. DOI: https://doi.org/10.1016/j.urology.2012.02.030

10. Giessing M. Transplant ureter stricture following renal transplantation: surgical options. DOI: https://doi. org/10.1016/j.transproceed.2010.12.014

11. Bachar GN, et al. Percutaneous balloon dilatation for the treatment of early and late ureteral strictures after renal transplantation: long-term follow-up DOI: 10.1007/ s00270-004-0163-9

12. Bromwich $\mathrm{E}$, et al. A 4-year review of balloon dilation of ureteral strictures in renal allografts. DOI: https://doi. org/10.1089/end.2006.20.1060

13. Aytekin C, et al. Percutaneous therapy of Ureteral obstructions andleak after renal transplantation: long-term results. Cardiovas Intervent Radiol 2007;30(6):1178-1184.

14. Routh J, et al. Urological complications and vesicoureteral reflux following pediatric kidney transplantation. DOI: https://doi.org/10.1016/j.juro.2012.09.091
15. Erturk E, et al. Treatment oftransplant ureteral stenosis with endoureterotomy. J Urol 1999;161(2):412-414.

16. Schwartz BF, et al. Treatment of refractory kidney transplantureteral strictures using balloon cautery endoureterotomy. Urology 20101;58(4):536-539.

17. Katz R, et al. Transurethral incisiónof ureteroneocystostomy strictures in kidney transplant recipients. BJU Int 2003;92(7):769-771.

18. Kristo B, et al. Treatment of renal transplant ureterovesical anastomoticstrictures using antegrade balloon dilation with or withoutholmium:YAG laser endoureterotomy. Urology 2003;62(5):831-834.

19. He Z, et al. Endoscopic incision for obstructionof vesicoureteric anastomosis in transplanted kidneys. BJU Int 2008;102(1):102-106.

20. Gdor Y, et al. Holmium: yttrium-aluminum-garnet laser endoureterotomy for the treatmentof transplant kidney ureteral strictures. DOI: 10.1097/TP.0b013e31816c7f19

21. Kumar $\mathrm{S}$, et al. Uretera obstruction following renal transplantation: causes, diagnosis and management. DOI: https://doi.org/10.1259/bjr.20140169

\section{AVISO IMPORTANTE}

La Revista Mexicana de Urología se convierte en una publicación solo digital, con todas las ventajas que los medios y dispositivos electrónicos ofrecen. Usted podrá revisar la información mediante el sitio web (www.revistamexicanadeurologia.org.mx) o descargando la app para Android o iPhone.

Para consultar el texto completo de los artículos deberá registrarse por una sola vez con su correo electrónico, crear una contraseña, indicar su nombre, apellidos y especialidad.

Esta información es indispensable para saber qué consulta y cuáles son sus intereses, y poder en el futuro inmediato satisfacer sus necesidades de información. 